\title{
The challenge of diabetic foot care: Review of the literature and experience at Queen Elizabeth Central Hospital in Blantyre, Malawi
}

\author{
Marianne M. Kasiya ${ }^{1}$, Grieves D. Mang'anda ${ }^{1}$, Sue Heyes ${ }^{2}$, Rejoice Kachapila ${ }^{3}$, Lydia Kaduya ${ }^{3}$, \\ Joy Chilamba ${ }^{3}$, Patrick Goodson ${ }^{3}$, Kondwani Chalulu ${ }^{3}$, Theresa J. Allain ${ }^{1}$
}

1. Department of Medicine, College of Medicine, University of Malawi, Blantyre, Malawi

2. Sheffield Teaching Hospitals NHS Trust, Sheffield, United Kingdom

3. Queen Elizabeth Central Hospital, Blantyre, Malawi

Correspondence: Dr Theresa J. Allain (theresa.allain@googlemail.com)

\begin{abstract}
Introduction
Diabetes mellitus is a group of metabolic diseases characterized by chronic hyperglycemia resulting from either inadequate insulin production, reduced tissue sensitivity to insulin or both. Chronic hyperglycaemia leads to diabetic complications including peripheral neuropathy, peripheral vascular disease, increased risk of infection and poor wound healing. The diabetic foot may be defined as a group of syndromes in which neuropathy, ischaemia, and infection lead to tissue breakdown resulting in morbidity and possible amputation. ${ }^{1}$ This review of the literature will cover the nature and epidemiology of diabetic foot disease and how it may be prevented and managed, with an emphasis on studies from Africa. The review will be illustrated with local experience of establishing a diabetic foot service at Queen Elizabeth Hospital in Blantyre.
\end{abstract}

\section{The global and local burden of diabetes}

Globally the prevalence of noncommunicable diseases (NCDs) is rising rapidly and it is predicted that they will become the leading cause of mortality worldwide in the next 30 years.

The global prevalence of diabetes in the adult population was $8.5 \%$ in $2014 .^{2}$ The greatest rate of increase will be in the developing countries of the world, including Malawi. Worldwide, deaths from diabetes are predicted to double between 2005 and 2030 and diabetic foot disease contributes significantly to this mortality.

In June 2010 the Malawi National STEPS study for noncommunicable diseases and their risk factors, described a countrywide survey of the prevalence of NCDs. In this, the prevalence of diabetes in adults aged 25 to 64 was estimated to be $5.6 \%,{ }^{3}$ revealing that diabetes is becoming a significant public health problem in Malawi.

A number of factors contribute to the increasing number of people with, mainly type 2, diabetes in Malawi. The country is undergoing a rapid social and demographic transition. Low birth weight is common and low birth weight predisposes to adult diabetes. ${ }^{4}$ Furthermore, HIV treatment and even HIV itself can lead to the metabolic syndrome, which leads to the development of diabetes or can worsen the glycaemic control of patients with existing diabetes. ${ }^{5}$ Since Malawi has a very high prevalence of HIV this is likely to impact on the prevalence of diabetes especially since, with free access to ART drugs, people with HIV are living longer.

\section{Diabetic foot ulcer overview}

Diabetic foot ulcer (DFU) is a major source of morbidity and a leading cause of hospitalization in patients with diabetes. ${ }^{6} \mathrm{It}$ is estimated that $50 \%$ of diabetes related hospital admissions are due to DFU. ${ }^{7}$ DFU can lead to infection, gangrene, amputation, and even death if necessary care is not provided. Overall, the rate of lower limb amputation in patients with $\mathrm{DM}$ is 15 times higher than patients without diabetes. It is estimated that approximately $50 \%-70 \%$ of all lower limb amputations are due to DFU. In addition, it is reported that every 30 seconds one leg is amputated due to DFU worldwide. $^{7}$ A study from Tanzania in 1997-8 found that 33\% of patients admitted with foot ulcers had amputations. The mortality rate was $29 \% .{ }^{8}$ Furthermore, DFU is responsible for substantial emotional and physical distress as well as productivity and financial losses that lower the quality of life. In the USA it was calculated that DFU accounted for $\$ 38.7$ billion of health expenditure in 2007. ${ }^{9}$ The cost of treating a diabetic foot ulcer in Tanzania was estimated at $\$ 3,060 .{ }^{10} \mathrm{In}$ many African countries, where resources are already scarce, diabetes imposes a heavy burden on already struggling health services and the financial burden to patients represents a greater proportion of household income.

\section{The burden of diabetic foot disease in sub-Saharan Africa}

Epidemiological surveys suggest that Africa has the second highest worldwide prevalence of DFU among diabetics of $7.2 \%(95 \% \mathrm{CI}: 5.1-9.3) .{ }^{11} \mathrm{~A}$ survey in the diabetic clinic at Queen Elizabeth Central Hospital (QECH), Blantyre in 2007, found that $8 \%$ of patients had foot ulcers or amputations and $6.7 \%$ had foot ulceration alone. There was a very low prevalence of arterial disease but peripheral neuropathy was present in 33\%. Of patients with neuropathy $12.1 \%$ had a foot ulcer and $4.5 \%$ had had an amputation compared to $4.1 \%$ and $0.09 \%$ in the non-neuropathy groups respectively, demonstrating that sensory loss was significantly associated with ulcers and amputation. ${ }^{12}$ The Malawi Diabetic Retinopathy Study (MDRS) of 357 diabetic subjects in 2012 found that $14.5 \%$ of its study subjects had neuropathy and $4.5 \%$ had foot ulcers of which $2.2 \%$ (8) had amputations. ${ }^{13}$ The results from these two studies illustrate that DFU is a problem amongst diabetic patients in Malawi.

The findings in Malawi are similar to other studies from the region. A study of 200 people attending a diabetes clinic 
in Ethiopia in 2014 found a prevalence of current or past foot ulcer of $15.5 \%$ with one amputation. ${ }^{14}$ Two studies from Cameroon, with data collected in 1999-2002 and 2006, showed prevalence of DFU of $10.7 \%$ among 503 patients and $13 \%$ among 300 patients respectively. ${ }^{15,16}$ A Tanzanian study of 474 patients seen during 1996-98 found that $10 \%$ had $\mathrm{DFU}^{17}$ and a study from South Africa showed that 6\% of 253 diabetic patients had some diabetic foot problem. ${ }^{18}$

In these studies the prevalence of peripheral neuropathy among all diabetics, whether or not they had DFU, was around 30\%. Peripheral vascular disease (PVD) was less common; in Cameroon the prevalence of PVD was $21 \%,{ }^{16}$ in Kenya $12 \%{ }^{19}$ in Tanzania $12.5 \%,{ }^{20}$ and in Malawi $7.5 \% .{ }^{12}$ A study from Kenya published in 2003, found that $47.5 \%$ of diabetic ulcers were neuropathic, $30.5 \%$ neuro-ischaemic and $18 \%$ ischaemic. $^{21}$

The associations of DFU have been described in Kenyan patients. The risk factors for DFU were longer duration of diabetes, poor glycaemic control, diastolic hypertension and poor self-care. ${ }^{20}$ Globally, a meta analysis of 9 studies, none from Africa, showed that DFU was significantly associated with older age, longer duration of DM, type $2 \mathrm{DM}$, lower $\mathrm{BMI}$, cigarette smoking, hypertension and the presence of retinopathy. ${ }^{11}$

In Malawi the prevalence of neuropathy and arterial disease, the presence of HIV and other diabetic complications has not been described in detail in prospective studies in patients with DFU. In addition the frequency with which a foot ulcer is the presenting symptom in a previously undiagnosed diabetic is not known, although the STEPS survey demonstrates that much diabetes remains undiagnosed. These are areas where further research is necessary.

\section{Management of diabetic foot ulcers}

DFU management needs a multidisciplinary approach. Often patients are in denial of their disease and fail to take ownership of their illness along with the necessary steps to prevent complications and to deal with the many challenges associated with the management of DFU.22 Management of DFU includes optimal blood glucose control, prevention through education, foot inspection and treatment.

\section{Prevention of diabetic foot ulcers: glycaemic control}

Good glycaemic control is important in preventing neuropathy and peripheral vascular disease; two of the important complications predisposing to DFU. The Diabetes Control and Complications Trial (DCCT) in type 1 DM showed that keeping blood sugar levels close to normal slows the onset and progression of eye, kidney and nerve diseases caused by diabetes. It demonstrated that any sustained lowering of blood sugar helps, even if the person has a history of poor control. ${ }^{23}$ The UK Prospective Diabetes Study (UKPDS) in type $2 \mathrm{DM}$ showed that good glycaemic control is important, not only for short term wellbeing of the patient but to prevent the long term complications. The endpoints of the studies were major clinical events which affect the life and well-being of patients, such as heart attacks, angina, strokes, amputations, blindness and renal failure. ${ }^{24}$ These studies also showed that good control of hypertension in diabetic patients was important in reducing long term complications. Prevention of diabetic foot ulcers: Screening and education Education of all diabetic patients about foot care is considered an essential part of every diabetic clinic, however, until recently the evidence to support this has been lacking. http://dx.doi.org/10.4314/mmj.v29i2.26
The Cochrane database review "Patient Education for Preventing Diabetic Foot Ulceration", published in 2014 concluded that although foot care education translated into better knowledge and positive short term behavior change there was a lack of studies that showed that this impacted on the important clinical endpoints of DFU and amputation. ${ }^{25}$ However, in 2015 an important paper was published that showed that, in high risk type 2 DM patients a 2 hour intensive education session led to a significant reduction in DFU over the next 6 months. ${ }^{26} \mathrm{~A}$ pioneering package of diabetes education, the Step by Step Foot Care Programme was established in Tanzania and is now used worldwide. ${ }^{27}$

Patients should be advised that meticulous attention to foot care and proper management of minor foot injuries are key to preventing ulcer formation. The need for daily feet inspection by the patient (or a caretaker), making an effort not to walk barefooted or get injuries to the feet, not to sit close to heaters or fires, wearing appropriate footware prevention of bathroom surgeries at home and avoidance of delays in presenting to hospital at the earliest onset of a foot lesion are the cornerstone of proper foot care. Gentle cleansing with soap and water, followed by the application of topical moisturizers or vaseline, helps to maintain healthy skin that can better resist breakdown and injury. Good resources for patient education can be found at http://iwgdf.org/step-bystep/.

In the clinic the physician or nurse should inspect the patient's shoes for areas of inadequate support or improper fit. Patients should choose the correct shoe size and appropriate footware (supportive shoes with an adequate toe box) to avoid ulcer formation and those with foot deformities or special support needs benefit from custom shoes. Foot inspection by clinic staff includes testing for neuropathy using a $10 \mathrm{~g}$ Semmes-Weinstein monofilament or $128 \mathrm{~Hz}$ tuning fork and peripheral vascular disease, ideally by measuring Ankle Brachial Pressure Indices (ABPIs) if foot pulses cannot be felt. Foot deformity and lesions, including minor lesions such as callus and tinea pedis, should be actively looked for and documented. A simple and quick diabetic foot assessment tool, designed to be completed in 60 seconds can be accessed at http://www.healthychange.ca/assets/files/ Inlow_Tool_2010.pdf. ${ }^{28}$ By reinforcing preventive advice and inspecting the patient's feet at routine follow-up visits, medical personnel can help the patient develop and maintain good foot-care habits. ${ }^{29,30}$

\section{Management of minor wounds}

Minor injuries and infections, such as cuts, scrapes, blisters and tinea pedis, can be unintentionally exacerbated by home remedies, for instance application of traditional medicine to wounds may delay healing. The use of iodine and eusol solutions for wound cleaning is not advised, but rather saline solutions should be used. Tinea pedis can be prevented by good foot hygiene and treated with topical antifungals such as miconazole or, if it is not available, gentian violet. Clinicians and nurses should inspect any minor wounds that do not heal rapidly.

\section{Callus removal}

Callus removal is another key step in preventing DFU. Callus (Figure 1) is an area of thick, dry skin that develops when skin is exposed to excessive pressure or friction due to pressure on bony areas of the foot, badly fitting shoes and dry skin. Calluses can lead to pressure necrosis of the underlying tissue and are often less sensitive to touch so if

Malawi Med J. 2017 Jun;29(2):218-223 


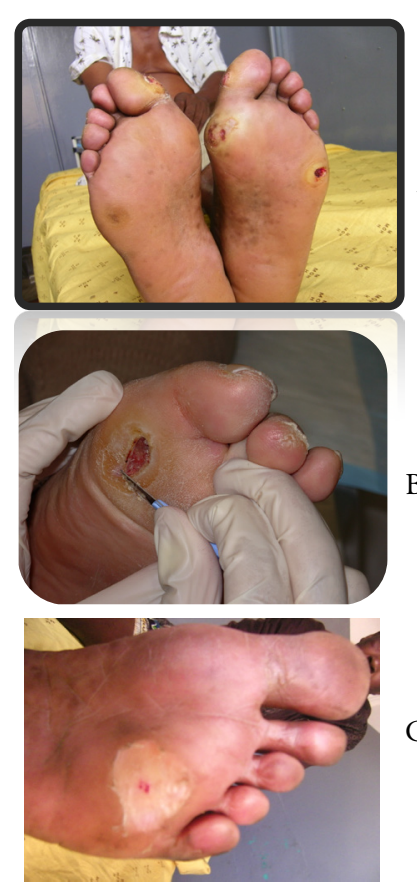

Figure 1: Examples of callus in diabetic foot

take longer to heal; they may also be colonized with bacteria, producing an unpleasant odor. These necrotic tissues may contain abscesses which can develop into general infection. It is therefore important to debride the wound in order to speed the healing process and avoid the need for amputation. A novel approach to debridement is the application of medicinal maggot therapy. This has been successfully described in a cohort of DFU patients in Egypt. ${ }^{31}$

\section{Antibiotics}

In Europe $58 \%$ of diabetic foot ulcers are infected at the time of first presentation. ${ }^{32}$ Infection rates from Africa are not known, but are likely to be similar or higher. Hence, antibiotic therapy is very important in the management of diabetic foot ulcer The initial antibiotic regime is usually selected empirically based upon clinical experience and local preferences; cover of gram positive cocci is essential. Antibiotics are modified on the basis of clinical response and wound culture/sensitivity results. Superficial wound swabs are often misleading as wounds are frequently colonised by non-pathogenic strains, only deep tissue samples, often obtained by debridement, are microbiologically useful.

Mild infection can be treated with oral antibiotics for 1 to 2 weeks using flucloxacillin, erythromycin, clindamycin, or amoxicillin/clavulanate. More severe infections require IV antibiotics for 2 to 4 weeks. If osteomyelitis is suspected 4-6 weeks treatment is needed. Antibiotics should provide broadspectrum cover directed toward staphylococcus aureus, streptococci, gram negative aerobes and anaerobic bacteria. More severe infections may require ceftriaxone, ciprofloxacin and metronidazole. Combinations of antibiotics may be needed to achieve adequate cover. Osteomyelitis should be excluded in all infected foot ulcers. A simple test is to pass a sterile probe into the base of the wound and if the probe touches straight to bone then osteomyelitis should be suspected. Not all osteomyelitis will be seen on a plain Xray but Xray of the foot should be performed. Figure 2 is an Xray showing osteomyelitis of the 5 th toe.

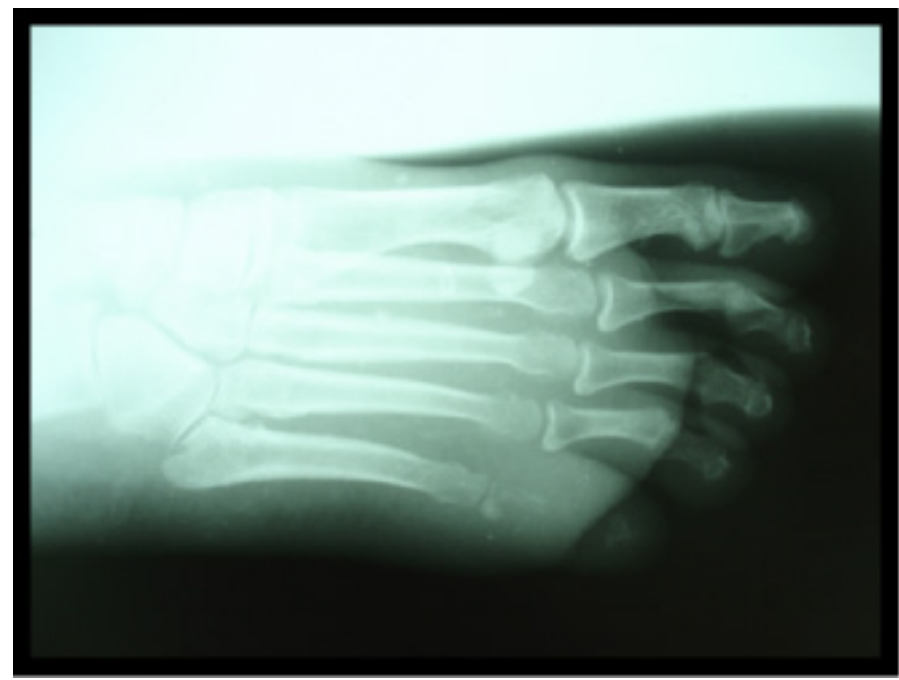

Figure 2: Foot $x$-ray showing osteomyelitis of the fifth toe

\section{Pressure relief through offloading}

In neuropathic ulcers abnormal pressures in the foot are a key factor in the development and impaired healing of DFU. Pressure can be offloaded through the use of plaster casts or bespoke footware where the insole is designed to remove pressure on points of ulceration. ${ }^{33}$ This technique can be applied even in resource poor settings through creative use of local materials.

\section{Amputation}

Major amputation (above or below knee) is a last resort and should almost never be needed to manage a diabetic foot ulcer. Debridement may require the removal of necrotic and infected tissue resulting in minor amputation of one or more toes or part of the forefoot but these will leave the patient with a viable foot on which they can still walk, avoiding the devastating personal and economic implications of amputation. This is especially important where artificial limbs are in poor supply for practical and economic reasons.

\section{Diabetes services in Malawi}

\section{Overview}

Historically diabetes has been thought to be uncommon in Malawi so diabetes services have not been high on the health agenda. Consequently, until recently Malawi had poorly organized diabetes care at district level, lack of knowledge and skills about diabetes care amongst health professionals and low awareness about diabetes and its complications in the general population. Many cases of diabetes go undiagnosed for years. Consequently, many are not identified and referred for treatment until it is too late - when the complications have become irreversible.

\section{Developments}

Over the past 7 years the College of Medicine (CoM) and the Ministry of Health $(\mathrm{MOH})$, with support from The World Diabetes Foundation (WDF), have been working together with the aim of improving services offered to diabetic patients in Malawi. Through staff training, a strong emphasis on patient education and the introduction of protocols these projects have strengthened the diabetes clinic at QECH and strengthened or established diabetes clinics at district and CHAM hospitals in Southern Malawi. Improvement in foot care has been a high priority in these developments. This has been supported by efforts towards gathering surveillance data and an electronic health record to address hypertension and diabetes management. ${ }^{34}$ Activities 


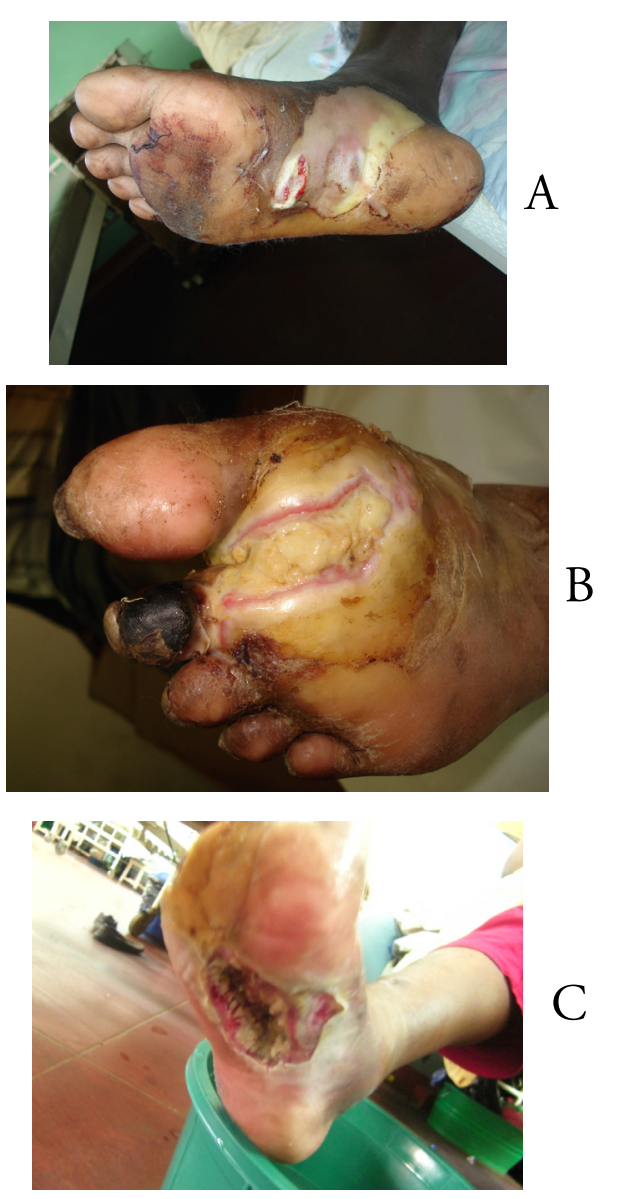

to enhance the national capacity for implementation of the diabetes and hypertension prevention and control strategies are now being scaled up to the rest of the country by the $\mathrm{MoH}$ in collaboration with Baobab Health Trust (BHT). Patient engagement has been ensured by close involvement of the Diabetes Association of Malawi (DAM). Structured group education, peer support, community awareness and education have been promoted by the DAM and Journalists Against Aids (JournAids).

\section{The experience of enhanced foot care at Queen Elizabeth Central Hospital (QECH)}

Education and awareness on foot care, for both staff and patients, has been included as part of general diabetes care at QECH since 2009. In 2011 a doctor with expertise in diabetic foot care joined the team at QECH. Under her guidance a specialist diabetic foot service evolved, which included a separate foot clinic. Clinicians and nurses who work in the clinic have undergone foot care training in Tanzania. The emphasis of the clinic is on both prevention and management of foot ulcers. Staff are able to recognise the type of foot problem (neuropathic, neuro-ischaemic or ischaemic), determine what type of debridement is needed (preventative debridement of callus in neuropathic feet or debridement of necrotic tissue in established ulcers) and undertake this. Wounds are dressed, antibiotics prescribed and advice on footwareis given. Off loading by adapting the patients own shoes with foam insoles is undertaken. Patients are seen weekly until their ulcer has healed. Clinic data is recorded on a Foot Mastercard for each patient. In parallel, inpatients on the surgical ward with diabetic foot sores have been visited regularly and records kept on their problem and outcome.
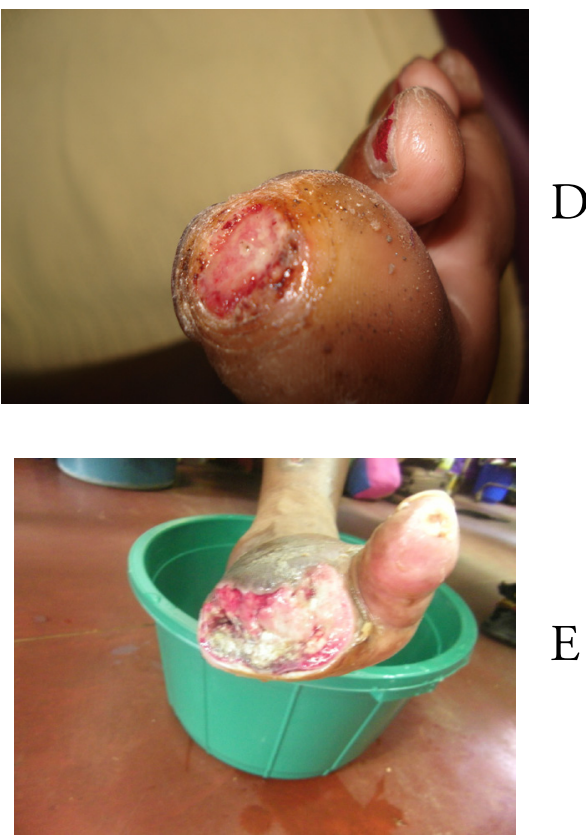

Figure 3: Examples of necrotic foot wounds managed at the Queen Elizabeth Central Hospital diabetic foot clinic

We have made major achievements in foot care. A number of feet have been salvaged through the established foot care clinic. Figure 3 shows examples of feet with necrotic wounds, all managed in the foot clinic. In all cases debridement, dressings and antibiotics resulted in the foot being salvaged leaving the patient with a functional foot which they could still walk on. Figure 4 shows before and after photographs of a patient from our clinic. The patient was told that he required below-knee amputation at the time photograph "a" was taken. Figure $4 \mathrm{~b}$ illustrates the improvement in the foot after local debridement, antibiotics and regular wound dressing. Rates of amputation have reduced; in 2010 eighteen
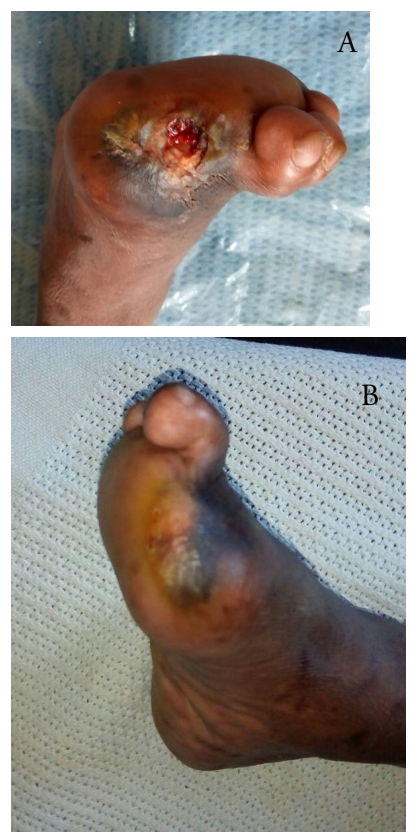

Figure 4: Necrotic wound that was (A) initially planned for management by below-knee amputation but (B) healed well after local debridement, antibiotics, and regular wound dressing

patients and in 2011 fifteen patients underwent major amputation for diabetic foot ulcers at QECH. Over the first 18 months of the foot clinic there were only 11 major amputations. Table 1summarizes the findings in 106 patients attending the diabetic foot clinic. The data was collected between December 2014 and May 2016. The age of patients 
Table 1: Diabetic foot ulcer type, treatment, and outcomes for patients attending the diabetic foot clinic at Queen Elizabeth Hospital

\begin{tabular}{|c|c|c|c|c|c|}
\hline Diagnosis & Total & Treatment plan & Total & Outcome & Total \\
\hline \multirow{6}{*}{ Neuropathic ulcer } & \multirow{6}{*}{46} & Wound dressing only & 28 & Normal healing & 16 \\
\hline & & Incision and drainage & 3 & Transferred out & 1 \\
\hline & & Debridement & 13 & Continued care & 16 \\
\hline & & Below knee amputation & 1 & Death & 0 \\
\hline & & Above knee amputation & 1 & Lost to follow-up & 13 \\
\hline & & Antibiotic & 19 & & \\
\hline \multirow{6}{*}{ Ischaemic ulcer } & \multirow{6}{*}{23} & Wound dressing only & 4 & Normal healing & 3 \\
\hline & & Incision and drainage & 1 & Transferred out & 0 \\
\hline & & Debridement & 10 & Continued care & 5 \\
\hline & & Below knee amputation & 5 & Death & 3 \\
\hline & & Above knee amputation & 3 & Lost to follow-up & 12 \\
\hline & & Antibiotic & 8 & & \\
\hline Neuropathic and ischaemic ulcer & 1 & Below knee amputation & 1 & Lost to follow-up & 1 \\
\hline \multirow{6}{*}{$\begin{array}{l}\text { Wound caused by trauma/injury/road } \\
\text { traffic accident }\end{array}$} & \multirow{6}{*}{27} & Wound dressing only & 20 & Normal healing & 10 \\
\hline & & Incision and drainage & 0 & Transferred out & 0 \\
\hline & & Debridement & 7 & Continued care & 0 \\
\hline & & Below knee amputation & 0 & Death & 1 \\
\hline & & Above knee amputation & 0 & Lost to follow-up & 16 \\
\hline & & Antibiotic & 4 & & \\
\hline Callus & 9 & Callus removal & 9 & Normal healing & 9 \\
\hline
\end{tabular}

ranged between 25 to 80 years with a mean of 55 years. The patients had a mean duration of diagnosis at 7.6 years. Amputations were more common in patients with DFU due to ischaemia, which is well recognized, as ischaemic wounds have impaired healing. These amputation rates are still higher than we would wish, mainly due to late presentations.

\section{Future prospects and recommendations}

\section{Challenges of managing diabetic foot ulcer in resource poor settings}

A leading diabetic foot specialist from our region has identified that the reasons for poor outcomes of foot complications in less-developed countries include: lack of awareness of foot care issues among patients and health care providers; shortage of trained health care workers for general diabetes care or specialist treatment; nonexistent podiatry services; shortages of medications and dressings, long distances for patients to travel to the clinic; delays among patients in seeking timely medical care, or among untrained health care providers in referring patients with serious complications for specialist opinion; lack of the concept of a team approach; absence of training programs for health care professionals; and finally lack of surveillance activities. ${ }^{10}$ He has also pointed out that there are ways of improving diabetic foot disease outcomes that do not require an exorbitant outlay of financial resources. These include implementation of sustainable training programmes for health care professionals, focusing on the management of the complicated diabetic foot and educational programmes that include dissemination of information to other health care professionals and patients. These proposals are clearly outlined in the highly successful Step by Step programme. ${ }^{35}$ We believe that we are making significant in-roads into preventing diabetic foot complications through a nurseled foot care clinic and ongoing training programmes for clinicians and nurses on preventing, screening and managing diabetic foot complications. Nationally the Ministry of Health needs to strengthen the undergraduate and postgraduate curricula for doctors, nurses and clinical officers so that management of all noncommunicable diseases, including diabetes is part of mainstream clinical education. Resources need to be deployed so that holistic diabetes care, including patient education and peer support is available at all levels of health facility and tertiary hospitals have the staff and facilities to manage the more severe complications of diabetes $w$ ith the ultimate aim to bring amputation rates to zero.

\section{Acknowledgements}

We would like to acknowledge the support of the World Diabetes Foundation, Copenhagen, Denmark who have provided financial support to our endeavours to improve diabetic foot care in Malawi. Further information can be found at: World Diabetes Foundation. Diabetes care in Southern Malawi WDF09-451 http://www. worlddiabetesfoundation.org/projects/malawi-wdf09-451, World Diabetes Foundation. Developing and sustaining comprehensive diabetes care in Malawi WDF13-842 http:// www.worlddiabetes foundation.org/projects / malawiwdf13-842, World Diabetes Foundation. Diabetes and Hypertension Control in Malawi WDF14-938 http://www. worlddiabetesfoundation.org/projects/malawi-wdf14-938.

\section{Competing interests}

All authors declare that they have no competing interests related to this work. 


\section{References}

1. Markakis K, Bowling FL, Boulton AJ. The diabetic foot in 2015: an overview, Diabetes Metab Res Rev. 2016; 32 Suppl 1:169-78

2. WHO fact sheet: Diabetes. Geneva, World Health Organization http:// www.who.int/mediacentre/factsheets/fs312/en/ Accessed 16.03.2017

3. Msyamboza K, Mvula CJ, Kathyola D. Prevalence and correlates of diabetes mellitus in Malawi: population-based national NCD STEPS survey. BMC Endocrine Disorders. 2014;14(1):41.

4. Hales CN, Barker DJP, Clark PMS, Cox LJ, Fall C, Osmond C, Winter PD. Fetal and Infant growth and impaired glucose tolerance at age 64. BMJ 1991; 303:1019-22

5. Tien P, Schneider M, Cole S et al. Antiretroviral therapy exposure and insulin resistance in the Women's Interagency HIV study. J Acquir Immune Defic Syndr 2008; 49: 369-76.

6. Joint British Diabetes Societies for Inpatient Care (JBDS - IP) December 2013 http://www.diabetologists-abcd.org.uk/JBDS/JBDS IP_Admissions_Avoidance_Diabetes.pdf Accessed 19.1.2016

7. Agwu E, Dafiewhare EO, Ekanem PE. (2011). Possible Diabetic-Foot Complications in Sub-Saharan Africa, Global Perspective on Diabetic Foot Ulcerations, Dr. Thanh Dinh (Ed.), ISBN: 978-953-307-727-7, InTech, DOI: 10.5772/29227.Available from: http://www.intechopen. $\mathrm{com} /$ books/global-perspective-on-diabetic-foot-ulcerations/possiblediabetic-foot-complications-in-sub-saharan-africa. Accessed 17.4.2015

8. Gulam-Abbas Z, Lutale JK, Morbach S, Archibald LK. Clinical outcome of diabetes patients hospitalized with foot ulcers, Dar es Salaam, Tanzania. Diabetic Medicine,19, 575- 579

9. Boulton AJ, Vileikyte L, Ragnarson-Tennvall G, Apelqvist J. The global burden of diabetic foot disease. Lancet. 2005 Nov 12;366(9498):1719-24.

10. Cavanagh P, Attinger C, Abbas ZG, Bal A, Rojas N, Xu ZR. Cost of treating diabetic foot ulcers in five different countries. Diabetes Metab Res Rev. 2012; 28: 107-111.

11. Zhang P, Lu J, Jing Y, Tang S, Zhu D, Bi Y. Global epidemiology of diabetic foot ulceration: a systematic review and meta-analysis. Ann Med. 2017 Mar;49(2):106-116.

12. Cohen DB, Allain TJ, Glover S et al. A Survey of the Management, Control, and Complications of Diabetes Mellitus in Patients Attending a Diabetes Clinic in Blantyre, Malawi, an Area of High HIV Prevalence. American Journal of Tropical Medicine and Hygiene. 2010 Sep $1 ; 83(3): 575-81$

13. Burgess P. Determinants of severity and progression of diabetic retinopathy in Southern Malawi. PhD thesis. 2016, The University of Liverpool, Foundation Building, Brownlow Hill, Liverpool, L69 7ZX, UK

14. Mamo T, Yifter H, Lemessa T. Risk factors assessment of diabetic foot ulcer using the sixty seconds screening tool: a hospital based crosssectional study at tikur anbessa specialized hospital. Ethiop Med J. 2015 Jul;suppl 2:45-9.

15. Kengne AP, Choukem SP, Dehayem YM, Simo NL, Fezeu LL, Mbanya JC. Diabetic foot ulcers in Cameroon: can microflora prevalence inform probabilistic antibiotic treatment? J Wound Care. 2006 Sep;15(8):363-6.

16. Ndip EA, Tchakonte B, Mbanya JC. A study of the prevalence and risk factors of foot problems in a population of diabetic patients in Cameroon. Int J Low Extrem Wounds. 2006 Jun;5(2):83-8.

17. Neuhann HF, Warter-Neuhann C, Lyaruu I, Msuya L. Diabetes care in Kilimanjaro region: clinical presentation and problems of patients of the diabetes clinic at the regional referral hospital-an inventory before structured intervention. Diabet Med. 2002 Jun;19(6):509-13

18. Rotchford AP, Rotchford KM. Diabetes in rural South Africa--an assessment of care and complications. S Afr Med J. 2002 Jul;92(7):53641.
19. Mugambi-Nturibi E, Otieno CF, Kwasa TO, Oyoo GO, Acharya K. Stratification of persons with diabetes into risk categories for foot ulceration. East Afr Med J. 2009 May;86(5):233-9.

20. Wikblad K, Smide B, Bergström A, Kessi J, Mugusi F. Outcome of clinical foot examination in relation to self-perceived health and glycaemic control in a group of urban Tanzanian diabetic patients. Diabetes Res Clin Pract. 1997 Sep;37(3):185-92.

21. Nyamu P M, Otieno CF, Amayo EO, McLigeyo SO. Risk factors and prevalence of diabetic foot ulcers at Kenyatta National Hospital, Nairobi. East Afr Med J. 2003 Jan;80(1):36-43.

22. Abbas ZG, Archibald LK. Challenges for management of the diabetic foot in Africa: doing more with less. International wound journal. 2007 Dec; 4(4):305-313.

23. Factors in development of diabetic neuropathy. Baseline analysis of neuropathy in feasibility phase of Diabetes Control and Complications Trial (DCCT). The DCCT Research Group. Diabetes, 1988 Apr; 37(4):476-81.

24. UK Prospective Diabetes Study (UKPDS). VIII. Study design, progress and performance. Diabetologia. 1991 Dec; 34(12):877-90.

25. Dorresteijn JA, Kriegsman DM, Assendelft WJ, Valk GD. Patient education for preventing diabetic foot ulceration. Cochrane Database Syst Rev. 2010 May 12;(5):CD001488. doi: 10.1002/14651858. CD001488.pub3.

26. Monami M, Zannoni S, Gaias M, Nreu B, Marchionni N, Mannucci E. Effects of a Short Educational Program for the Prevention of Foot Ulcers in High-Risk Patients: A Randomized Controlled Trial. Int J Endocrinol. 2015;2015:615680. doi: 10.1155/2015/615680. Epub 2015 Sep 10. Accessed 20th March 2017

27. The International Working Group on the Diabetic Foot (IWGDF). Step by Step foot care for people with diabetes http://iwgdf.org/step-bystep/ Accessed 20th March 2017

28. Inlow S. A 60 second foot exam for people with diabetes. Wound Care Canada. 2004;2(2):10-1

29. Armstrong DG, Lavery LA. Diabetic foot ulcers: prevention, diagnosis and classification. Am Fam Physician, 1998 Mar 15; 57(6):1325-32, 1337-8.

30. Sibbald RG, Mayer D, Goodman L et al. Diabetic foot ulcers: Part II. Management. J Am Acad Dermatol. 2014; 70:21.e1-2124.

31. Tantawi TI, Gohar YM, Kotb MM, Beshara FM, El-Naggar MM. Clinical and microbiological efficacy of MDT in the treatment of diabetic foot ulcers. J Wound Care. 2007 Oct;16(9):379-83.

32. Prompers L, Schaper N, Apelqvist J, et al. Prediction of outcome in individuals with diabetic foot ulcers: focus on the differences between individuals with and without peripheral arterial disease. The EURODIALE Study. Diabetologia 2008; 51(5): 747-55.

33. Bus SA, Armstrong DG, van Deursen RW, Lewis J, Caravaggi CF, Cavanaghon PR on behalf of the International Working Group on the Diabetic Foot (IWGDF). IWDGF Guidance on footware and offloading interventions to prevent and heal foot ulcers in patients with diabetes 2015 http://www.iwgdf.org/files/2015/website_footwearoffloading.pdf Accessed 20th March 2017

34. Allain TJ, Oosterhout JJ, Douglas GP, Joukes S, Gadabu OJ, Darts C, Kapur A, Harries AD. Applying the "DOTS" Tuberculosis Model to monitoring and evaluating persons with diabetes mellitus in Blantyre, Malawi. 2011 Tropical Med Int Health 2011 Sep;16(9):1077-84

35. Bakker K, Abbas ZG, Pendsey S. Step by Step, improving diabetic foot care in the developing world. A pilot study for India, Bangladesh, Sri Lanka and Tanzania Pract Diab Int 2006; 23(8):1-5. 